\title{
Rapid method for Mycobacterium tuberculosis identification using electrospray ionization tandem mass spectrometry analysis of mycolic acids
}

Diagnostic Microbiology and Infectious Disease 76 (2013) 298-305

http://dx.doi.org/10.1016/j.diagmicrobio.2013.03.025

\section{Szewczyk Rafał ${ }^{a, *}$, Kowalski Konrad $^{b}$, Janiszewska-Drobinska Beata ${ }^{c}$, Druszczyńska Magdalena $^{d}$}

a," Corresponding author - Department of Biotechnology and Industrial Microbiology, Institute of Microbiology, Biotechnology and Immunology, Faculty of Biology and Environmental Protection, University of Łódź, Banacha 12/16, 90-237 Łódź, Poland, tel. 48426354460, Fax. 484266558 18, rszewcz@biol.uni.lodz.pl

“'Neolek" Laboratory of Biomedical Chemistry, Department of Experimental Oncology, Institute of Immunology and Experimental Therapy, PAS, Rudolf Weigl 12, 53-114 Wrocław, Poland ' Specialized Hospital of Tuberculosis, Lung Diseases and Rehabilitation, Szpitalna 5, 95-080 Tuszyn, Poland

${ }^{\mathrm{d} D e p a r t m e n t ~ o f ~ I m m u n o l o g y ~ a n d ~ I n f e c t i o u s ~ B i o l o g y, ~ I n s t i t u t e ~ o f ~ M i c r o b i o l o g y, ~ B i o t e c h n o l o g y ~ a n d ~ I m m u n o l o g y, ~ F a c u l t y ~ o f ~ B i o l o g y ~ a n d ~ E n v i r o n m e n t a l ~}$ Protection, University of Łódź, Banacha 12/16, 90-237 Łódź, Poland

\section{Abstract}

Mycolic acids, which play a crucial role in the architecture of mycobacterial cell walls, were analyzed using electrospray ionization tandem mass spectrometry (ESI-MS/MS). A targeted analysis based on the 10 most abundant and characteristic multiple reaction monitoring (MRM) pairs was used to profile the crude fatty acid mixtures from $M$ tb and several nontuberculous mycobacterial strains. Comparative analysis yielded unique profiles for mycolic acids, enabling the reliable identification of mycobacterial species. In a case-control study of TB and non-TB Polish patients, we demonstrated the potential diagnostic utility of our approach for the rapid diagnosis of active TB with sensitivity and specificity surpassing those of existing methods. This robust method allows the identification of TB-positive patients after $2 \mathrm{~h}$ of sample preparation in the case of direct sputum analysis or 10 days of culturing, both of which are followed by one minute of LC-

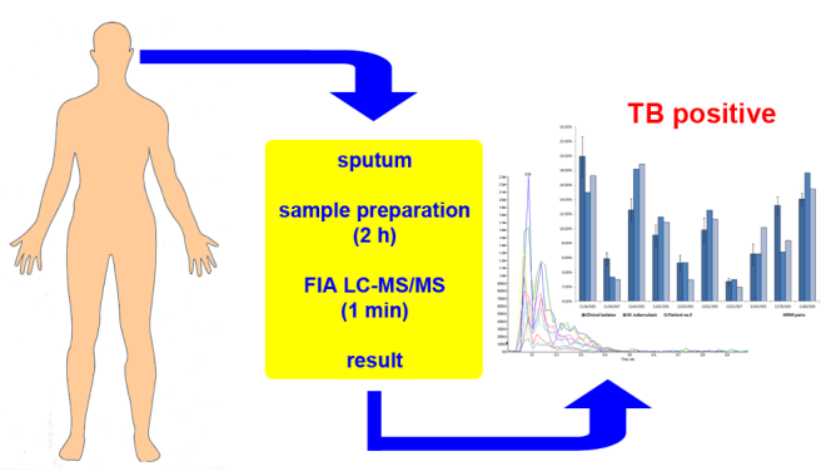
MS/MS analysis.

Keywords: Mycobacterium tuberculosis, mycolic acids, mass spectrometry, liquid chromatography, multiple reaction monitoring

\section{Introduction}

Mycobacterium tuberculosis (Mtb) is an intracellular pathogen and the causative agent of tuberculosis (TB). Despite the discovery of the bacilli over 100 years ago and the availability of effective drugs for over 50 years, the disease still remains one of the most lifethreatening infectious diseases on earth. In 2011, there were an estimated 8.7 million cases of TB (13\% co-infected with HIV) and 1.4 million deaths as a result of TB infection (WHO, 2012). Furthermore, one third of the world's population is latently infected with Mtb, which remains the largest reservoir of the tubercle bacilli.

In the absence of an effective TB vaccine, early diagnosis and treatment of the active disease remains the most important part of TB control programs. TB diagnosis methods, which were developed over 100 years ago, have not changed significantly for many decades. AFB smear microscopy, the oldest diagnostic method, detects only approximately $50 \%$ of active TB cases but still remains the most widely used test in the world. Mtb culture, the other diagnostic gold standard, is more sensitive than sputum smears but takes 2-6 weeks to produce a result (Dorman, 2012). The currently available tests based on DNA amplification or interferon- $\gamma$ release are expensive and require specialized equipment and qualified staff (Drobniewski et al., 2003; Schuluger, 2001; Vittor et al., 2011). The lack of accurate, rapid and cheap methods for both diagnosis and drug susceptibility testing leaves large numbers of patients undetected and greatly hinders global TB control (Ottenhoff et al., 2012).

The cell walls of $M t b$, along with those of other members of the Mycobacterium genus, have a unique composition. Mycolic acids play a crucial role in the architecture of their cell envelope; these acids are high-molecular-weight $\alpha$-alkyl- $\beta$-hydroxy fatty acids of exceptional length and complexity that are unique to mycobacteria and related acid-fast bacteria (Brennan and Nikaido, 1995; Takayama et al. 2005; Yuan et al., 1998). In Mtb, they are characterized by very hydrophobic $\mathrm{C}_{54}$ to $\mathrm{C}_{63}$ fatty acids with $\mathrm{C}_{22}$ to $\mathrm{C}_{24} \alpha$ side chains (Fujiwara et. al 2012; Takayama et al. 2005). They are attached to penta-arabinose termini of arabinogalactan and provide a lipophilic anchorage for a variety of waxes and glycolipids. Mycolic acids comprise up to $30 \%$ of the mycobacterial cellular dry weight and are actively synthesized during the vegetative growth of bacteria. Most mycobacterial species contain a combination of different types of mycolic acids, and the mycolate composition of mycobacteria has been used extensively for taxonomic purposes. Differences in the length and chemical structure of mycolic acids isolated from different species suggest their possible use as sensitive biomarkers of mycobacterial infections.

Over the past 20 years, much progress has been made in the separation and molecular characterization of mycobacterial mycolic acids. One of the techniques recommended by the Center for Disease Control (CDC) is high-performance liquid chromatography (HPLC)(CDC, 1996). However, this method is quite sophisticated and expensive relative to other laboratory techniques and is not commonly used in many reference laboratories. Moreover, it requires multiple pretreatment steps for lipid purification and a constant retention time for accurate results (Butler and Guthertz, 2001) More precise methods of mycolic acid analysis involve rapid, high-throughput mass spectrometry techniques that produce both qualitative and quantitative results (Shui et al., 2012; Song et al. 2009).

In this work, we focused on the development of a rapid ESI-MS/MS technique for the identification and confirmation of TB infection on a triple-quadrupole instrument equipped with an ESI ion source and standard HPLC system. To our knowledge, this study is one of the first to apply ESI-MS/MS for the detection and recognition of $M$. tuberculosis mycolic acids directly in human sputum. This method is rapid and sensitive due to its accurate MRM transitions for the selected MAs and reduced sample consumption and preparation time.

\section{Materials and methods}

\subsection{Reagents, solvents and standards}

The analysis presented in this work required high-purity reagents and solvents. For the LC-MS/MS mobile phase, an acetonitrile (J.T. Baker), methanol (Sigma-Aldrich), chloroform (J.T. Baker) and $1 \mathrm{M}$ ammonium formate (Sigma-Aldrich) solution in water (Milli-Q, Millipore) was used. The compounds used for the mycolic acid preparation were concentrated $\mathrm{HCl}$ (Polish Chemical Reagents S.A. $\mathrm{POCH}), \mathrm{Na}_{2} \mathrm{SO}_{4}$ anhydrous (POCH) and $\mathrm{KOH}(\mathrm{POCH})$. A mycolic acid standard from Mycobacterium tuberculosis $\mathrm{H}_{37} \mathrm{R}_{\mathrm{v}}$ (M4537) was purchased from Sigma-Aldrich.

\subsection{Bacterial/fungal strains and culturing conditions}

M. tuberculosis $H_{37} R_{v}$ strain (ATCC 27294), M. bovis, 12 Mtb clinical isolates (Clinical Division of Pulmonology and Alergology, Medical University of Łódź, Poland) and the non-tuberculous mycobacterial strains (MOTT) $M$. avium, $M$. intracellulare, $M$. abscessus and $M$. kansasii (Polish Collection of Microorganisms (PCM), Polish Academy of Sciences, Wrocław, Poland) were cultivated in 7H9 Middlebrook broth (Difco Laboratories Ltd., West Molesey, UK) supplemented with $0.05 \%$ Tween 80 (Sigma) at $37^{\circ} \mathrm{C}$ under rotating conditions $(125 \mathrm{rpm})$ until the mid-logarithmic phase. Bacterial and fungal strains (Corynebacterium glutamicum, Rhodococcus equi, Rhodococcus erythropolis, Proteus vulgaris, Escherichia coli, Listeria innocua, 
Klebsiella pneumoniae, Arthrobacter sp., Pseudomonas fluorescens, Aspergillus nidulans, Cunninghamella elegans, Metarhizium anisopliae, Schizosaccharomyces pombe, Saccharomyces cerevisiae) from the collection of Institute of Biotechnology, Microbiology and Immunology, University of Lodz, Poland were cultivated as a liquid cultures in glucose broth medium at $37^{\circ} \mathrm{C}$ (bacteria) or Sabouraud broth in $28^{\circ} \mathrm{C}$ (fungi and yeasts). Bacterial $\mathrm{CFU} / \mathrm{ml}$ were determined by plating several dilutions on Middlebrook $7 \mathrm{H} 10$ agar supplemented with OADC (mycobacteria), glucose agar (bacteria) or Sabouraud agar (fungi and yeasts) and counting the colonies appearing after $24 \mathrm{~h}$ (bacteria), 5-7 days (fungi) or 6 weeks (mycobacteria) of incubation at $37^{\circ} \mathrm{C}$ or $28^{\circ} \mathrm{C}$ depending on the tested microorganism.

\subsection{Demographics and clinical descriptions of the subjects}

This study analyzed 44 sputum samples collected from 44 BCGvaccinated HIV-negative participants. Of these subjects, 16 were adult patients with active pulmonary TB (12 men and 4 women) recruited from the Specialized Hospital of Tuberculosis, Lung Diseases and Rehabilitation in Tuszyn, Poland. In $13(81 \%)$ cases, active TB was confirmed by sputum smear microscopy for acid-fast bacilli or the bacteriological isolation of $M$. tuberculosis. Three further TB cases were diagnosed based on radiological findings compatible with active TB, clinical symptoms and responses to anti-TB treatment. The sputum samples were collected before or during the first 2 weeks of treatment. As controls, 17 adult non-TB patients $(12$ men and 5 women), hospitalized with pulmonary symptoms but eventually diagnosed with pulmonary diseases other than TB (11 bacterial pneumonia, 3 lung cancer, 3 bronchitis), along with 11 healthy controls ( 5 men and 6 women) were included in the study. None of these individuals had any known history of contact with TB and showed negative TB bacteriology. The sputum collection and the study followed the ethical guidelines of the Specialized Hospital of Tuberculosis, Lung Diseases and Rehabilitation in Tuszyn, Poland.

\subsection{Sputum processing}

Sputum specimens $(2 \mathrm{ml})$ were mixed with equal volumes of $\mathrm{NaOH}-\mathrm{N}$ acetyl cysteine solution $(4 \% \mathrm{NaOH}, 1 \% \mathrm{~N}$-acetyl cysteine) and left at $37^{\circ} \mathrm{C}$ for $30 \mathrm{~min}$ with occasional vortexing. Subsequently, $4 \mathrm{ml}$ of $2.9 \%$ sodium citrate and $8 \mathrm{ml}$ of sterile $0.067 \mathrm{M}$ phosphate buffer $(\mathrm{pH} 6.8)$ were added. After centrifugation (3500 rpm, $20 \mathrm{~min}$ ), the supernatants were decanted and the pellets were used for (1) the preparation of smears for standard Ziehl-Neelsen staining, (2) direct extraction of mycolic acids for LC-MS/MS analysis, (3) inoculation into 7H9 Middlebrook broth supplemented with $0.05 \%$ Tween 80 and incubation for 10 days at $37^{\circ} \mathrm{C}$ (indirect LC-MS/MS analysis) and (4) inoculation onto Löwenstein-Jensen agar slants and culture for 4-6 weeks at $37^{\circ} \mathrm{C}$. When colonies were grossly observed on the surface of the Löwenstein-Jensen medium, M. tuberculosis species were confirmed and verified using acid-fast staining and niacin testing.

\subsection{Mycolic acid extraction from bacteria and human sputum}

The extraction procedure was based on previously published methods using alkali hydrolysis (CDC, 1996; Butler and Guthertz, 2001). The initial mycolic acid isolation procedure involved 18 modifications including the influence of cell pretreatment (surface lipids methanol extraction and removal), alkali hydrolysis ( $\mathrm{KOH}$ concentration, temperature, incubation time) as well as the MAs extraction procedure (extraction solvent, volume, time and number of repeats). Every modification was checked multiple times to determine its repeatability and reproducibility. The optimized method was significantly more sensitive and robust than the initial method and consisted of the following steps: a Mycobacterium cell suspension $\left(10^{7} \mathrm{cells} / \mathrm{ml}\right)$ or preprepared sputum were transferred into a twisted glass vial and centrifuged ( $3500 \mathrm{rpm} ; 10 \mathrm{~min} ; 4^{\circ} \mathrm{C}$ ). The obtained cell sediment was re-suspended in $2 \mathrm{ml}$ of methanol, shaken on vortex for $30 \mathrm{~s}$ and centrifuged again as described above. The methanol was then decanted from the cell sediment and the cells were re-suspended in 2 $\mathrm{ml}$ of $25 \% \mathrm{KOH}$ in methanol. The bacterial cell suspensions were incubated at $90^{\circ} \mathrm{C}$ for $1 \mathrm{~h}$ in tightly closed glass vials. After cooling to room temperature and acidification with $1.5 \mathrm{ml}$ of concentrated $\mathrm{HCl}$ $(38 \%)$, the samples were extracted 3 times with $2 \mathrm{ml}$ of chloroform (30 $\mathrm{s}$ on vortex, $3000 \mathrm{rpm})$. Water aliquots were removed from the extracts by the addition of anhydrous sodium acetate and evaporated to dryness under a vacuum evaporator. The dry residue was dissolved in $1 \mathrm{ml}$ of chloroform or LC-eluent and transferred to HPLC vials for LC-MS/MS analysis.

\section{6. $L C-M S / M S$ analysis}

Flow injection analysis (FIA) was used to detect and profile the mycolic acids. The FIA-LC-MS/MS analyses were performed on an Agilent 1200 LC system coupled with an AB Sciex 3200 QTRAP mass detector. The liquid chromatography parameters were as follows: injection volume, $10 \mu \mathrm{l}$; draw speed, $200 \mu \mathrm{l} / \mathrm{min}$; eject speed and eluent flow, $400 \mu \mathrm{l} / \mathrm{min}$. The mobile phase consisted of methanol:chloroform:acetonitrile (20:40:40) with the addition of $5 \mathrm{mM}$ ammonium formate. The needle was washed for $3 \mathrm{~s}$ in chloroform in the flush port after every injection. The mass detector was set to a negative ionization MRM mode with an ESI (Turbo V) ion source. The optimized ion source parameters were as follows: curtain gas, 10; IS, $4500 \mathrm{~V}$; temperature, $650{ }^{\circ} \mathrm{C}$; GS1, 50; GS2, 65. The optimized compound-dependent parameters for 10 MRM are shown in table 1. The entire method involves $1 \mathrm{~min}$ of analysis time. The injection cycle applied for system cleaning and conditioning consisted of the following injections and method runs: $100 \mu \mathrm{l}$ of chloroform, $10 \mu \mathrm{l}$ of the blank and $10 \mu \mathrm{l}$ of the tested sample.

\subsection{Statistical analysis}

The average intensity of the MRM signals for the 10 mycolic acids listed in the Table 1 was calculated by a chromatogram averaging from 0.01 to $0.3 \mathrm{~min}$ of the LC run. Each MRM pair percentage was calculated as the percent of the intensity related to the corresponding MRM sum of the tested sample (100\% intensity). Statistical analyses were performed using the calculated intensity percentages transformed using the arcus sinus function. These values directly correspond to the composition percentage of all 10 chosen mycolic acids.

To confirm homogeneity of each mycolic acid content within the pattern isolated from $M t b$ species and extracted from sputum samples, Student's t-test for the comparison of two means was performed. Average intensities for each mycolic acid content were firstly transformed by arcus sinus function for all samples in both groups: $M t b$ species $(n=12)$ and extracted sputum samples $(n=16)$. Then average and standard deviation values inside groups were calculated and compared using Student's t-test for the comparison of two means. The presented results are confidence interval $95 \%$ of differences between two means for each analyzed mycolic acid separately.

\section{Results}

\subsection{Mycobacterial mycolic acids profiles}

The ESI-MS/MS conditions were optimized using a mycolic acid standard from a virulent Mtb strain (M4537, Sigma-Aldrich). Initially, we focused on the analysis of the fragmentation pattern of the MAs by Q1 scans, precursor ion scans, product ion scans and $\mathrm{MS}^{3}$ scans to confirm the structure of the MA chains postulated by other authors (Figure 1). The most significant data came from the product ion and $\mathrm{MS}^{3}$ experiments (Figure $1 \mathrm{C}$ and $1 \mathrm{D}$ ). Fragments coming from the cleavage of C-C bond between carboxyl $\alpha$-alkyl chain and hydroxyl meromycolic chain in the range of 300 to $430 \mathrm{~m} / \mathrm{z}$ are most intensive and characteristic fragmentation pattern for MAs. To confirm the C-C cleavage between the MAs chains, we focused on the $600-900 \mathrm{~m} / \mathrm{z}$ region, where we found very weak but detectable fragments coming from the corresponding meromycolic chains.

Table 1. Most of the characteristic mycolic acid MRM pairs chosen for diagnostic purposes and their MS/MS compound-dependent parameters: Q1 - precursor mass, Q2 - product ion mass, Time - dwell time (ms), DP - declustering potential, EP - entrance potential, CEP - collision cell entrance potential, CE - collision energy, CXP - collision cell exit potential.

\begin{tabular}{ccccccccccc}
\hline $\begin{array}{c}\text { Mycolic } \\
\text { acid form }\end{array}$ & $\begin{array}{c}\text { Chemical } \\
\text { formula }\end{array}$ & $\begin{array}{c}\text { Q1 } \\
\text { mass } \\
{[\mathrm{m} / \mathrm{z}]}\end{array}$ & a-branch & $\begin{array}{c}\mathrm{Q} 3 \text { mass } \\
{[\mathrm{m} / \mathrm{z}]}\end{array}$ & $\begin{array}{c}\text { Time } \\
(\mathrm{ms})\end{array}$ & DP & EP & CEP & CE & CXP \\
\hline $\boldsymbol{\alpha}-$ & $\mathrm{C}_{78} \mathrm{H}_{152} \mathrm{O}_{3}$ & 1136.4 & $\mathrm{C}_{24}$ & 395.4 & 40 & -155 & -9.5 & -84 & -84 & -3 \\
$\alpha-$ & $\mathrm{C}_{78} \mathrm{H}_{152} \mathrm{O}_{3}$ & 1136.4 & $\mathrm{C}_{22}$ & 367.3 & 40 & -155 & -9.5 & -84 & -74 & -3 \\
$\alpha-$ & $\mathrm{C}_{80} \mathrm{H}_{156} \mathrm{O}_{3}$ & 1164.4 & $\mathrm{C}_{24}$ & 395.4 & 40 & -155 & -9.5 & -60 & -86 & -3 \\
$\alpha-$ & $\mathrm{C}_{82} \mathrm{H}_{160} \mathrm{O}_{3}$ & 1192.3 & $\mathrm{C}_{24}$ & 395.4 & 40 & -155 & -10 & -48 & -88 & -3 \\
$\alpha-$ & $\mathrm{C}_{84} \mathrm{H}_{164} \mathrm{O}_{3}$ & 1220.3 & $\mathrm{C}_{24}$ & 395.4 & 40 & -155 & -10 & -66 & -82 & -3 \\
methoxy- & $\mathrm{C}_{85} \mathrm{H}_{168} \mathrm{O}_{4}$ & 1252.3 & $\mathrm{C}_{24}$ & 395.4 & 40 & -155 & -9 & -72 & -82 & -3 \\
methoxy- & $\mathrm{C}_{85} \mathrm{H}_{168} \mathrm{O}_{4}$ & 1252.3 & $\mathrm{C}_{22}$ & 367.3 & 40 & -155 & -9 & -72 & -80 & -3 \\
keto- & $\mathrm{C}_{86} \mathrm{H}_{168} \mathrm{O}_{4}$ & 1264.4 & $\mathrm{C}_{24}$ & 395.4 & 40 & -160 & -9 & -34 & -84 & -3 \\
keto- & $\mathrm{C}_{87} \mathrm{H}_{177} \mathrm{O}_{4}$ & 1278.4 & $\mathrm{C}_{24}$ & 395.4 & 40 & -160 & -9.5 & -50 & -84 & -3 \\
methoxy- & $\mathrm{C}_{87} \mathrm{H}_{172} \mathrm{O}_{4}$ & 1280.4 & $\mathrm{C}_{24}$ & 395.4 & 40 & -160 & -10 & -54 & -88 & -3 \\
\hline
\end{tabular}

As an example, figure $1 \mathrm{C}$ shows the fragmentation of $\alpha-\mathrm{MA}$ (1164.6 $\mathrm{m} / \mathrm{z}, \mathrm{C}_{80} \mathrm{H}_{153} \mathrm{O}_{3}$ ), where the peaks for the $\mathrm{C}_{26}, \mathrm{C}_{24}$ and $\mathrm{C}_{22}$ carboxyl $\alpha$ alkyl chains are at $395.5 \mathrm{~m} / \mathrm{z}, 367.5 \mathrm{~m} / \mathrm{z}$ and $339.4 \mathrm{~m} / \mathrm{z}$, respectively, and those for the $\mathrm{C}_{54}, \mathrm{C}_{56}$ and $\mathrm{C}_{58}$ meromycolic chains are at 768.2 $\mathrm{m} / \mathrm{z}, 796.5 \mathrm{~m} / \mathrm{z}$ and $824.4 \mathrm{~m} / \mathrm{z}$, respectively. Similar fragmentation schemes were observed in all tested MAs (data not shown), including methoxy- and keto- forms. Further fragmentation of the base peaks ( $\alpha$ - 

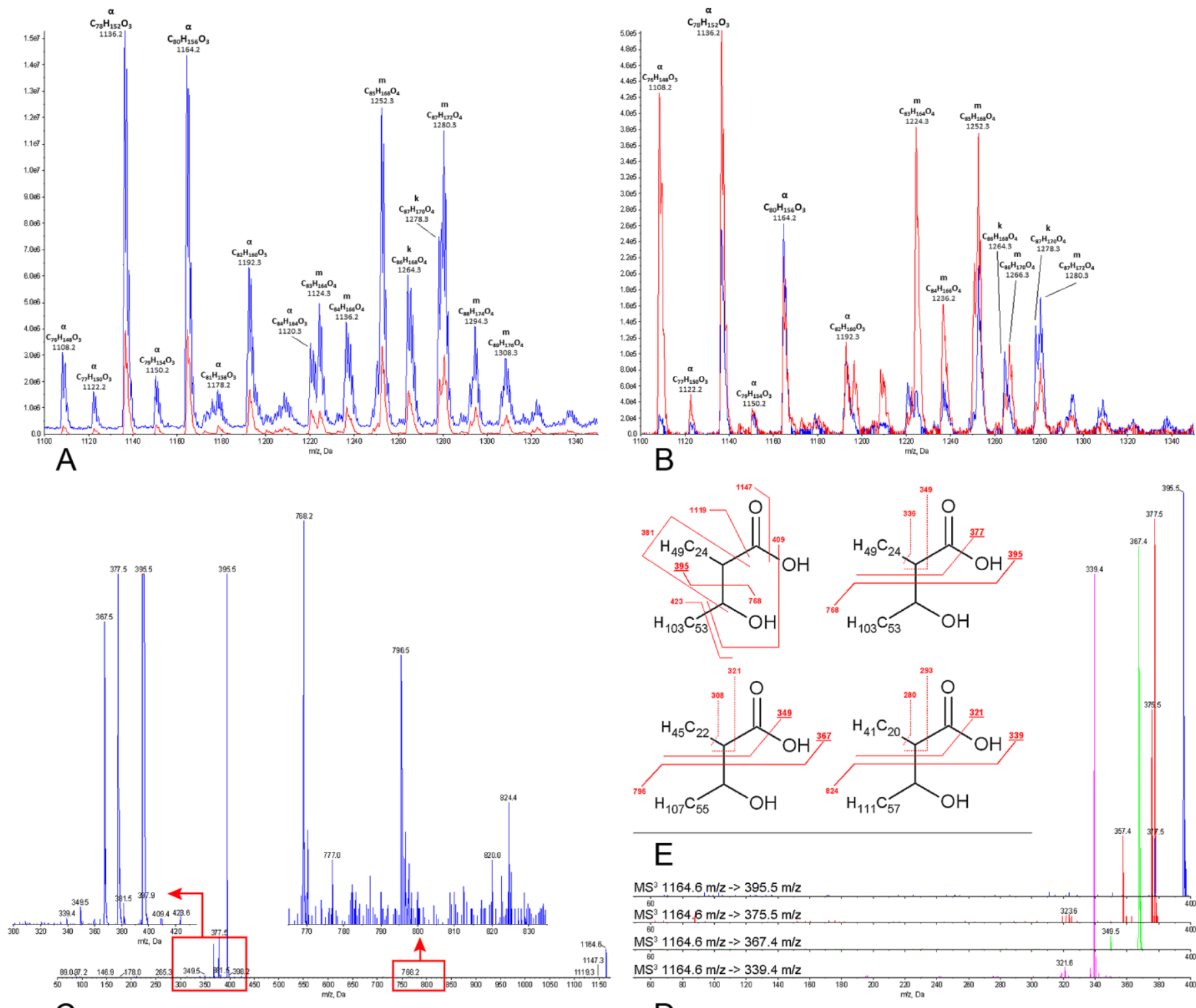
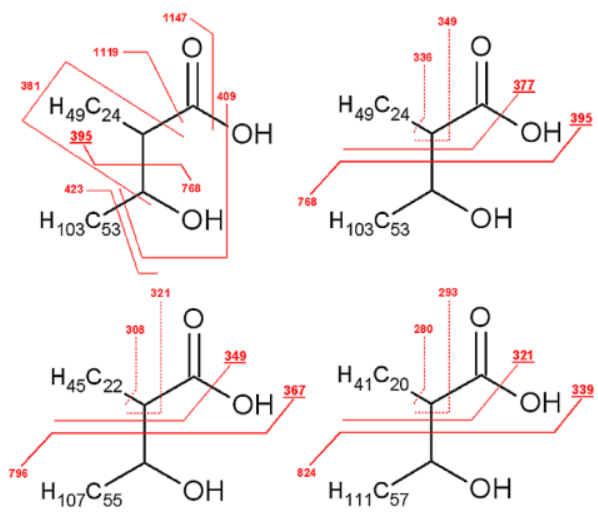

$\mathrm{E}$

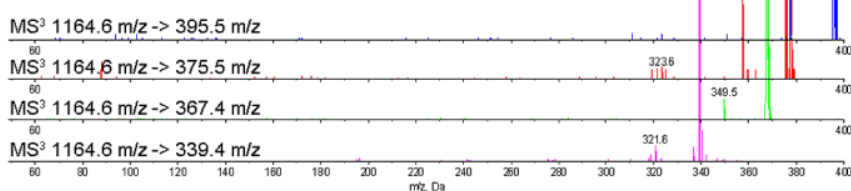

\section{D}

The potential matrix effect on the mycolic acid extraction and identification was evaluated by analyzing three samples: (1) the sputum of a healthy control volunteer, (2) the sputum of a healthy control volunteer inoculated in vitro with $10^{8} \mathrm{M}$. tuberculosis $\mathrm{H}_{37} \mathrm{R}_{\mathrm{v}}$ cells $/ \mathrm{ml}$, (3) $10^{8}$ cells $/ \mathrm{ml}$ suspension of $M$. tuberculosis $\mathrm{H}_{37} \mathrm{R}_{v}$ prepared in 7H9 Middlebrook medium. The results indicated no influence of the matrix on the intensity and profile of the mycolic acids using the applied extraction and LC-MS/MS method.

Further analysis focused on the ability of the tested method to differentiate between Mycobacterium species. As shown in table 2, the mycolic acid profiles of MOTT strains are significantly different from those of $M t b \mathrm{H}_{37} \mathrm{R}_{\mathrm{v}}$ and $M$. bovis BCG strains. Two MRM transitions, $1136 / 367$ and $1252 / 367$, are the most specific for the identification of the selected MOTT species because these species feature $\mathrm{C}_{24}$-long $\alpha$ alkyl chains, whereas the other analyzed mycolic acids include $\mathrm{C}_{26^{-}}$ long $\alpha$-alkyl chains. Within the tested MOTT strains, the mycolic acid profile can be used to identify $M$. avium and $M$. kansasii not $M$. abscessus and $M$. intracellulare, as there are no significant differences in the mycolic acid profiles of these strains.

Studies of the local Mtb population were performed by comparative analysis of the clinical Mtb isolates and TB patients. Statistical analysis of the difference in the average intensity for each mycolic acid content in the two groups was based on Student's t-test for the comparison of the two means. Mean differences in each mycolic acid content in one group were subtracted from the values for the tested mycolic acid in the second group. Statistical significance of differences is shown as confidence intervals $95 \%(\mathrm{Cl} 95)$ for the comparison of the two means. Cl95, which does not include 0 , represents a statistically significant difference between two groups. As presented in Figure 2, a significant difference was observed only in case of three compounds (1252/367, 1264/395, 1220/395 MRM pairs), which highlights the possible range of characteristic mycolic acid profiles for all $M t b$ strains. Despite the slight differences in the percentage of individual mycolic acids from clinical $M t b$ strains or the virulent reference $\mathrm{H}_{37} \mathrm{R}_{v}$ strain, a 
Mtb "fingerprint" is still easily identified and distinguished (Figure 3). Unfortunately, the data do not allow M. bovis BCG and M. tuberculosis strains to be distinguished.

The extraction of mycolic acids was also performed from bacteria within Corynebacterineae suborder not belonging to the Mycobacterium genus, in which shorter-chain mycolic acids naturally occur in the cell wall as well as bacteria not belonging to the Corynebacterineae suborder, selected microscopic fungi and yeasts. The obtained results supported the high specificity of the applied LC MS/MS method, with no false positive results for any of the tested extracts from the selected strains (Table S-1 in Supplementary material).

Table 2. Mycolic acid profiles of $\mathrm{Mtb}$ and Ntm reference strains

\begin{tabular}{ccccccc}
\hline \multirow{2}{*}{$\begin{array}{c}\text { Mycolic } \\
\text { acids }\end{array}$} & \multicolumn{3}{c}{ Mtb } & \multicolumn{3}{c}{ Ntb } \\
\cline { 2 - 7 } & $\begin{array}{c}M \text {. } \\
\text { tuberculosis }\end{array}$ & $\begin{array}{c}\text { M. bovis } \\
\text { BCG }\end{array}$ & $\begin{array}{c}M \text {. } \\
\text { kansasii }\end{array}$ & $\begin{array}{c}M \text {. } \\
\text { avium }\end{array}$ & $\begin{array}{c}\text { M. } \\
\text { intracellulare }\end{array}$ & $\begin{array}{c}M \text {. } \\
\text { abscessus }\end{array}$ \\
\hline $1136 / 395$ & $14.96 \%$ & $16.50 \%$ & $0.53 \%$ & $0.47 \%$ & $0.07 \%$ & $0.37 \%$ \\
$1136 / 367$ & $3.30 \%$ & $3.32 \%$ & $53.74 \%$ & $29.60 \%$ & $98.41 \%$ & $97.67 \%$ \\
$1164 / 395$ & $18.17 \%$ & $20.21 \%$ & $1.11 \%$ & $2.38 \%$ & $0.26 \%$ & $0.43 \%$ \\
$1192 / 395$ & $11.60 \%$ & $11.25 \%$ & $1.13 \%$ & $12.17 \%$ & $0.15 \%$ & $0.22 \%$ \\
$1220 / 395$ & $5.35 \%$ & $4.47 \%$ & $0.45 \%$ & $6.16 \%$ & $0.18 \%$ & $0.09 \%$ \\
$1252 / 395$ & $12.58 \%$ & $13.17 \%$ & $0.67 \%$ & $0.97 \%$ & $0.29 \%$ & $0.22 \%$ \\
$1252 / 367$ & $2.99 \%$ & $2.86 \%$ & $39.44 \%$ & $35.53 \%$ & $0.32 \%$ & $0.19 \%$ \\
$1264 / 395$ & $6.53 \%$ & $8.14 \%$ & $0.28 \%$ & $2.45 \%$ & $0.00 \%$ & $0.15 \%$ \\
$1278 / 395$ & $6.84 \%$ & $0.40 \%$ & $1.81 \%$ & $8.01 \%$ & $0.11 \%$ & $0.19 \%$ \\
$1280 / 395$ & $17.68 \%$ & $19.69 \%$ & $0.85 \%$ & $2.24 \%$ & $0.19 \%$ & $0.47 \%$ \\
\hline
\end{tabular}

\subsection{Clinical sample analysis}

LC-MS/MS analysis of 16 sputum samples obtained from TB patients indicated the presence of Mtb mycolic acids in $11(69 \%)$ specimens directly or $15(94 \%)$ specimens after 10 days of incubation. In contast, the presence of AFB bacilli was confirmed in 9 out of $16(56 \%)$ TB patients, whereas M.tb culture was positive for $13(81 \%)$ specimens. No signal was measured in the sputum from non-TB patients or healthy controls, suggesting that this method is very specific with no cross-reactivity. Using our method we also detected mycolic acids of $M$ tb in a 10-day incubated sputum from TB patients with negative smear and culture results (material no. 13, 14, 15). However, we observed one false negative result in a patient with confirmed TB (material no.16), suggesting that our method needs improvement in terms of mycolic acid extraction efficiency or instrument sensitivity (Table S-2 in Supplementary material). Examples of the raw LCMS/MS data are presented in figure 4.

\section{Discussion.}

Mycolic acid profiles can be used as a tool for the classification of the infecting mycobacteria (CDC, 1996; Butler and Guthertz, 2001, Shui et al., 2012); however, for our purposes, the most important was the fact that Mtb mycolic acid profile is unique and can be used as a diagnostic marker for TB confirmation or exclusion in clinical samples. On the other hand, the proper identification of MOTT species seems to be critical, as some species are known to be naturally resistant to one or more anti-TB drugs.

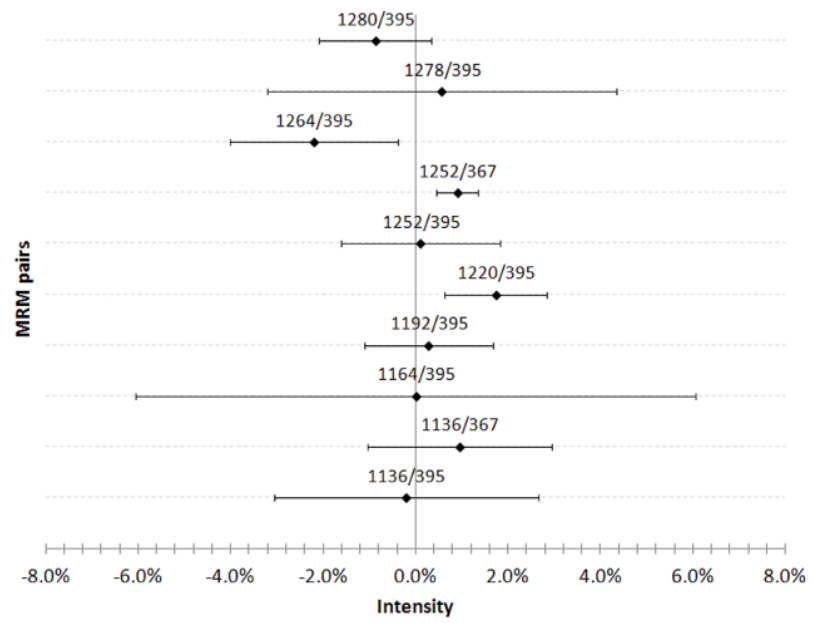

Fig. 2. Statistical analysis of differences in the MA content obtained from TB patients from extracted sputum $(n=16)$ and Mtb clinical isolates $(n=12)$. Figure presents mean results of the Student's t test for the comparison of the 2 means (groups) in the case of each MA separately with $\mathrm{Cl} 95$.

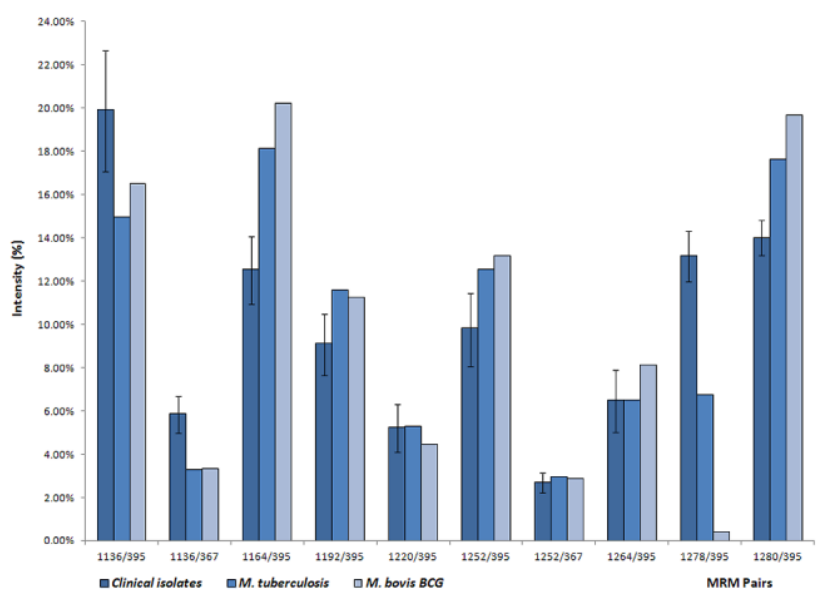

Fig. 3. Differences in MAs profiles between clinical isolates $(n=12)$ and reference strains of Mtb and M. bovis BCG. The values are the percentages of the average intensity for each MA to the total intensity of all analyzed compounds with a Cl95.

Mass spectra analyses presented in this work prove the structure of MAs postulated by other authors. Similar results and mass spectra interpretations based only on precursor ion scans were presented by Song et al., 2009 and Shui et al., 2012. The precursor ion scan (Figure $1 \mathrm{~A}$ and $1 \mathrm{~B}$ ) showed that the $\mathrm{C}_{26}$ chain is the dominant $\alpha$-alkyl chain in the mycolic acid profile of $M t b \mathrm{H}_{37} \mathrm{R}_{\mathrm{v}}$. Although there are many recognized species of Mycobacteria the domination of the $\mathrm{C}_{26}$ chain seems to be characteristic of Mtb which cause active TB in humans (Song et al., 2009) therefore it is possible to differentiate between $M$ tb and MOTT group only the basis of the a-mycolic chain length. In our method MRM pairs including a longer $\left(\mathrm{C}_{26}\right) \alpha$-mycolic chain ( $\mathrm{Q} 3$ mass - $395 \mathrm{~m} / \mathrm{z}$ ) were used to confirm the presence of Mtb while MRM pairs built on a shorter $\left(\mathrm{C}_{24}\right)$ a-mycolic chain determination (Q3 mass - 367 $\mathrm{m} / \mathrm{z}$ ) let us check the presence of MOTT strains. The differences between the 10 MRM pairs chosen for TB diagnostics in our study and the 14 chosen by Shui et al., 2012 are the use of 1220 and $1278 \mathrm{~m} / \mathrm{z}$ in our approach and 1196, 1224 and $1208 \mathrm{~m} / \mathrm{z}$ their approach. Additionally, we chose two Q3-367 m/z-based MRMs, whereas Shui et al., 2012 choose three of this type of MRM transitions for the final diagnostic method. The use of different mycolic acid patterns in the Mtb diagnostics in our work did not affect data acquisition and interpretation.

Mycolic acids are important part of the cell wall for a wide group of microorganisms within Corynebacterineae suborder. These compounds are characterized by a variety in size and structure of the aliphatic chains. As it was shown by Kowalski et al. (2012) the total carbon of MAs from Mtb covers the range 70-90 and only Tsukamurella genus has a potential for a cross-talk with $M$ tb species as it covers the range 64-78 in general. However, within the chosen 10 MRM pairs (Table 1) the lowest number of carbon atoms in the analyzed mycolic acid moiety is 78 . The only one environmental isolate without any clinical significance, described as $T$. carboxydivorans poses a similar range of the total carbon atoms (Park et al. 2009), but overlapped range of the total carbon atoms do not have to lead to the cross-signal as Tsukamurella genus is also characterized by highly unsaturated (up to 8 double bonds) meromycolic chain without any functional groups (Yassin et al. 1996). In the case of Mycobacterium there are three potential locations where double bonds could appear in alpha mycolic acids and also three places where functional groups could appear in keto- or metoxymycolic acids. Considering the double filtering of the $m / z$ in the MRM scan mode, MRM pairs selection in the final method and data presented in table S-1 (Supplementary material) we think it is highly unlikely to obtain false positive signals from other organisms than Mycobacterium.

Although mycolic acids are ideal conservative compounds for Mtb confirmation studies, some territory, culturing, time of growth or physiological state related differences in their compositions were observed. Experimental data demonstrated that mycolic acids from Mtb, consisting mainly of alpha-, keto-, and methoxy-mycolates, contain mixtures of homologs with different chain lengths or stereochemistries around the functional groups in the main chain (Beukes et al. 2010). These variables can possibly cause slight differences in the content of the single mycolic acid. However, variability could be omitted during analysis of the mycolic acid pattern consisting of 10 different mycolic acids - e.g. characteristic for Mycobacterium tuberculosis presence. Accurate and high-throughput analysis of mycolic acids patterns form clinical samples from all over the world as well as reference species collected in microorganisms 

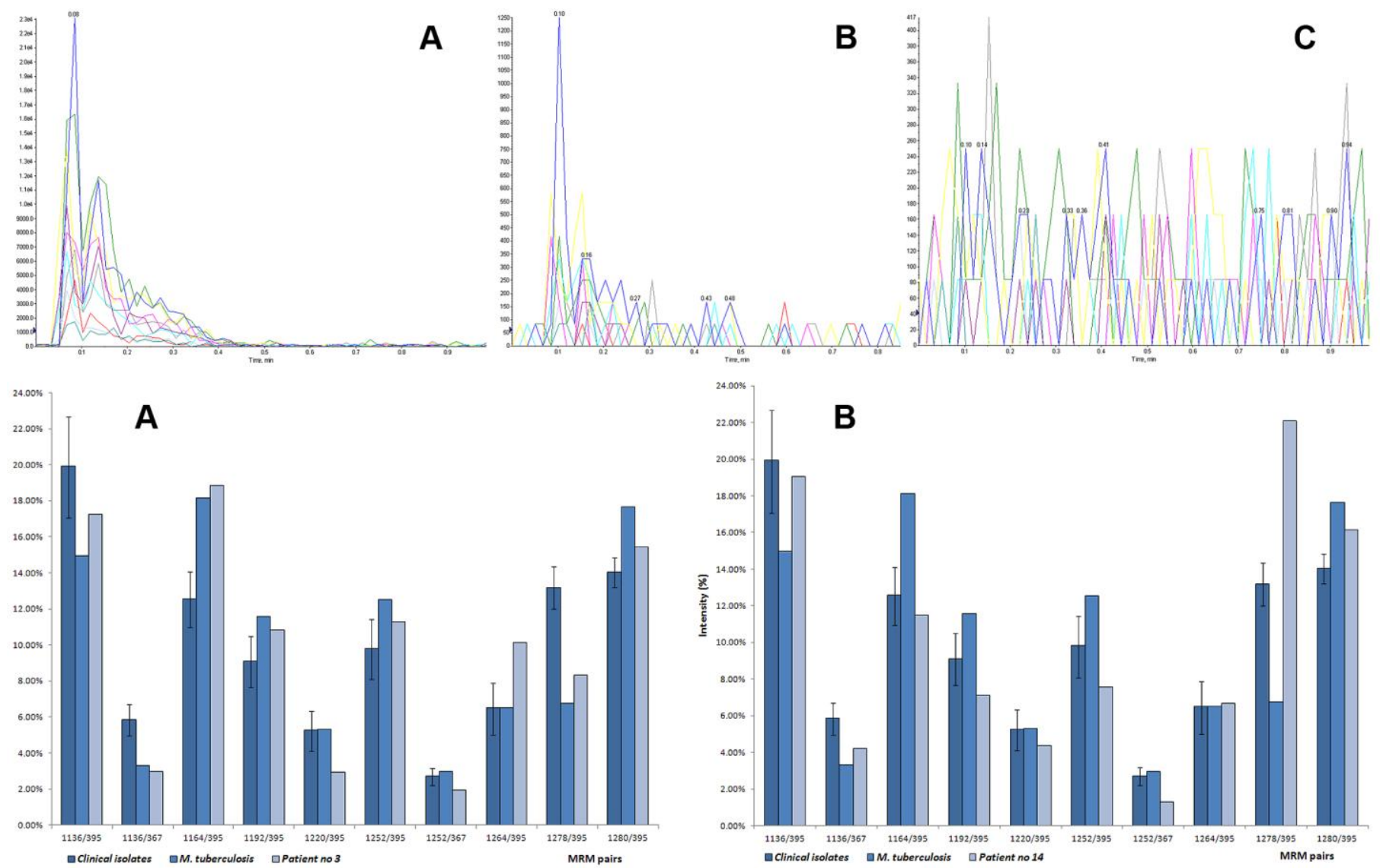

Fig. 4. Typical positive (A), unsure (B), and negative $(C)$ results for LC-MS/MS analysis.

banks could be the source of future results leading to a creation of global standards for mycolic acids patterns typical not only for Mtb but also for each species of atypical mycobacteria.

The diagnosis of TB infection or drug efficacy studies with mass spectrometry involve different approaches. Matrix-assisted laser desorption ionization time of flight mass spectrometry (MALDI-TOFMS) is used in proteomic or/and intact cell analysis. The MALDI-TOFMS whole proteome analysis conducted by Wang et al. showed that proteins are useful biomolecules for the differentiation of mycobacterial strains; however, this analysis is not highly specific because of the matrix proteins and noise issues. Similar methods were used by El Khéchine et al., 2011 and Shitikov et al., 2012 again, the targeted protein analysis was only applicable to previously cultured mycobacteria cells. Intact cell analysis by MALDI-TOF MS incorporates analysis of all compounds coming from the laser-ionized cell envelopes. A mass spectra fingerprint can be used for diagnostic purposes for mycobacterial cultures (Lotz et al., 2012; Saleeb et al. 2011). MS methods are robust, fast, cheap and effective but also require bacteria culturing, which can take between 1 day and 2 months depending on the tested species. Thus, they can be successfully applied in drug efficacy studies but are less desirable for diagnostic work. To our knowledge, the work published by Deng et al. is the first to use MALDI-TOF-MS to analyze clinical samples, but the developed method combines standard methods and MS/MS analysis. The authors analyzed blood samples with respect to the presence of amyloid $A$ and transthyretin using ELISA testing and neopterin and $C$ reactive proteins by MALDI-TOF-MS. The results from both tests were used for the estimation of active pulmonary TB. The test exhibited $85.7 \%$ sensitivity and $83.3 \%$ specificity. Sample preparation using weak cation ion exchange magnetic beads and the authors' suggestion to use 2-D electrophoresis instead make this method too complex for routine analysis.

Mycolic acids as a biomarker seem to be most appropriate for diagnostic purposes as they occur in high concentration in the cell walls of mycobacteria, regardless of growth conditions, and are relatively easy to extract. MS/MS analysis of the MA profiles in MRM mode is the most successful method for the TB diagnoses in patient sputum or cell cultures, as described in our work or by Shui et al., 2012. The presented results obtained using LC-MS/MS analysis are better than those obtained using the current TB diagnostic methods with one powerful advantage: the possibility of detecting and recognizing $M t b$ within the same day $(2 \mathrm{~h}$ for sample preparation and $1 \mathrm{~min}$ for LC-MS/MS analysis) or, at worst, 10 days from the start

of the test. In contrast, 3 to 6 weeks are required for the detectable growth of $M t b$ on solid media due to the long doubling time of the bacteria. Although acid-fast staining of clinical material remains the simplest and most frequently used microbiological test for TB detection, the major limitation is its poor sensitivity. Sputum smear examination for acid-fast bacilli allow diagnosing up to $50 \%$ of TB cases, whereas a sputum culture can confirm pulmonary TB in around $80 \%$ of true cases (Siddiqi et al., 2003). However, the detection of acid-fast bacilli in a sputum smear is not affirmative for $M t b$, because many nontuberculous mycobacteria may also lead to positive AFB staining results (Jafari et al., 2006). The overall sensitivity of the direct ESI-MS/MS analysis of mycolic acids (69\%) in our study was similar to the sensitivity of the sputum smear, however it was significantly improved after a 10-day incubation period (94\%). Using our method we detected Mtb mycolic acids also in the sputum of patients with negative bacteriological results. Although smear-negative patients are less infectious than patients with bacteriology confirmed TB, they also contribute to the transmission of TB (Behr et al., 1999). Light microscopy can detect mycobacteria in the sputum at a minimum density of 5000-10000 bacterial cells per $\mathrm{ml}$ of specimen, whereas the infectious dose is only a few mycobacterial cells. Therefore, people in contact with smear-negative TB patients are also at risk of $M$. th infection and subsequent development of active TB disease (Tostmann et al., 2008). However, it must be stated that these promising results should be confirmed on a larger group of TB patients with negative bacteriology results. We think that the technique might be also useful in the diagnosis of TB forms other than pulmonary, especially those in which it is not possible to detect mycobacteria using traditional diagnostic methods.

The MRM-based method developed by Shui et al., 2012 requires 2,5day sample preparation followed by rapid ( $2 \mathrm{~min}$ ) MS/MS analysis and exhibits $94 \%$ sensitivity and $93 \%$ specificity; however, only $25 \%$ of smear-negative patients produced positive MAs signals. In contrast, in our work, positive MAs signals were produced by $43 \%$ of the smearnegative patients in direct sputum analysis and $86 \%$ after 10 -day culturing. In both cases, positive bacterioscopy resulted in $100 \%$ confirmation by MS/MS methods regardless of the number of bacteria cells observed. Similar MAs extractions to those presented in this work were applied by Viader-Salvadó et al., 2007; however, the measurement of MAs was performed on HPLC with fluorescence detection during a 15 min gradient (methanol:chloroform) analysis with a $2.5 \mathrm{ml} / \mathrm{min}$ flow rate. The obtained results did not significantly extend the specificity or sensitivity relative to the standard AFB staining method.

Novel TB diagnosis methods involve genetic analysis of DNA or RNA (Daley et al., 2008; Toney et al., 2010). Xpert MTB/RIF assay is an 
example of a sensitive, specific and rapid PCR-based method (Chang et al., 2012; Lawn et al., 2011). The specificity of the test is $99 \%$; the sensitivity for smear-positive patients is in the range of $97.9-98.4 \%$, whereas that for bacterioscopy-negative patients is $73.1 \%$. This method is also used for rifampicin resistance tests. MTB/RIF assay is currently recommended by WHO for TB diagnosis.

The initial cost of the LC-MS/MS equipment is relatively high however, the per sample costs for our method are much less than those for DNA-based techniques because we use only common reagents for sample preparation and analysis, whereas molecular methods require expensive hybridization probes or primers. The estimated cost of the sample analysis (including only consumables and reagents) performed with our method is approximately $\$ 0.60$ per sample. The final cost of the test may vary depending on the country currency, personnel costs, equipment amortization and service, but the overall cost will likely be competitive with those of conventional and genetic methods. Considering the cost of the LC-MS/MS apparatus, genetic methods are preferred as a novel diagnostic tool however, with the current progress in clinical applications, an increasing number of laboratories are using MS/MS techniques in various tests. Presented results, although preliminary and based on a limited number of tested samples, look very promising and in the future studies should be done including more patients. We see a huge potential for the developed method in clinical laboratories or hospitals already having or planning to buy LC-MS/MS instrumentation as it can be applied not only for $M t b$ detection but also for other mycobacterial infection confirmations and drug efficacy studies.

\section{Conclusion}

In summary, a rapid, reliable, robust and relatively low-cost diagnostic technique developed by our group is an attractive alternative for rapid TB diagnosis, which is one of the most difficult challenges facing clinicians. It is estimated that each person with untreated TB infects an average of between 10 and 15 people every year, remaining a reservoir from which active TB can develop. Currently, conventional methods require an average of 3-6 weeks to obtain a result, which leads to delays in the introduction of effective treatments to stop the spread of $M$. tuberculosis in the environment and reduce the rate of transmission.

\section{Acknowledgments}

The authors wish to thank the Laboratory of Biology of Mycobacteria, Łódź, Poland for their contribution to the collection of clinical and microbiological data, useful discussion and practical help with sputum processing.

\section{References}

Behr MA, Warren SA, Salamon H, Hopewell PC, Ponce de Leon A, Daley CL, et al. Transmission of Mycobacterium tuberculosis from patients smear-negative for acid-fast bacilli. Lancet. 1999;353:444-9. Beukes M, Lemmer Y, Deysel M, Al Dulayymi JR, Baird MS, Koza G, et al. Structure-function relationships of the antigenicity of mycolic acids in tuberculosis patients. Chem Phys Lipids. 2010;163:800-8.

Brennan PJ, Nikaido $H$. The envelope of mycobacteria. Annu Rev Biochem 1995;64:29-63.

Butler WR, Guthertz LS. Mycolic acid analysis by high-performance liquid chromatography for identification of Mycobacterium species. Clin Microbiol Rev 2001;14:704-26.

Centers for Disease Control and Prevention. Standardized Method for HPLC Identification of Mycobacteria, 1996.

Chang K, Lu W, Wang J, Zhang K, Jia S, Li F, et al. Rapid and effective diagnosis of tuberculosis and rifampicin resistance with Xpert MTB/RIF assay: a meta-analysis. J Infect 2012;64:580-8.

Daley P, Petrich A, May K, Luinstra K, Rutherford C, Chedore P, et al. Comparison of in-house and commercial 16S rRNA sequencing with high-performance liquid chromatography and genotype AS and CM for identification of nontuberculous mycobacteria. Diagn Microbiol Infect Dis 2008;61:284-93.

Deng C, Lin M, Hu C, Li Y, Gao Y, Cheng X, et al. Exploring serological classification tree model of active pulmonary tuberculosis by magnetic beads pretreatment and MALDI-TOF MS analysis. Scand J Immunol 2011;74:397-405.

Dorman SE. New diagnostic tests for tuberculosis: bench, bedside and beyond. Infect Dis Clin 2010;50:173-7.

Drobniewski FA, Caws M, Gibson A, Young D. Modern laboratory diagnosis of tuberculosis. Lancet Infect Dis 2003;3:141-7.

El Khéchine A, Couderc C, Flaudrops C, Raoult D, Drancourt $M$. Matrix-assisted laser desorption/ionization time-of-flight mass spectrometry identification of mycobacteria in routine clinical practice. PLoS One 2011;6:e24720.
Fujiwara N, Naka T, Ogawa M, Yamamoto R, Ogura H, Taniguchi H. Characteristics of Mycobacterium smegmatis J15cs strain lipids. Tuberculosis (Edinb) 2012;92:187-92.

Jafari C, Ernst M, Kalsdorf B, Greinert U, Diel R, Kirsten D, et al. Rapid diagnosis of smear-negative tuberculosis by bronchoalveolar lavage enzyme-linked immunospot. Am J Respir Crit Care Med 2006;174:1048-54

Kowalski K, Szewczyk R, Druszczyńska M. Mycolic acids - potential biomarkers of opportunistic infections caused by bacteria of the suborder Corynebacterineae. Postepy. Hig. Med. Dosw. 2012;66:461 8.

Lawn SD, Nicol MP. Xpert® MTB/RIF assay: development, evaluation and implementation of a new rapid molecular diagnostic for tuberculosis and rifampicin resistance. Future Microbiol 2011;6:106782.

Lotz A, Ferroni A, Beretti JL, Dauphin B, Carbonnelle E, Guet-Revillet $\mathrm{H}$, et al. Rapid identification of mycobacterial whole cells in solid and liquid culture media by matrix-assisted laser desorption ionization-time of flight mass spectrometry. J Clin Microbiol 2010;48:4481-6.

Ottenhoff TH, Ellner JJ, Kaufmann SH. Ten challenges for TB biomarkers. Tuberculosis (Edinb) 2012;92:S17-20.

Park SW, Kim SM, Park ST, Kim YM. Tsukamurella carboxydivorans sp. nov., a carbon monoxide-oxidizing actinomycete. Int J Syst Evol Microbiol 2009;59:1541-44.

Saleeb PG, Drake SK, Murray PR, Zelazny AM. Identification of mycobacteria in solid-culture media by matrix-assisted laser desorption ionization-time of flight mass spectrometry. J Clin Microbiol 2011;49:1790-4.

Schluger NW. Changing approaches to the diagnosis of tuberculosis. Am J Respir Crit Care Med 2001;164:2020-4

Shitikov E, Ilina E, Chernousova L, Borovskaya A, Rukin I, Afanas'ev $\mathrm{M}$, et al. Mass spectrometry based methods for the discrimination and typing of mycobacteria. Infect Genet Evol 2012;12:838-45.

Shui G, Bendt AK, Jappar IA, Lim HM, Laneelle M, Hervé M, et al. Mycolic acids as diagnostic markers for tuberculosis case detection in humans and drug efficacy in mice. EMBO Mol Med 2012;4:27-37.

Shui G, Bendt AK, Pethe K, Dick T, Wenk MR. Sensitive profiling of chemically diverse bioactive lipids. J Lipid Res 2007;48:1976-84.

Siddiqi K, Lambert M-L, Walley J. Cical diagnosis of smear-negative pulmonary tuberculosis in low-income countries: the current evidence. Lancet Infect Dis 2003;3:288-96.

Song SH, Park KU, Lee JH, Kim EC, Kim JQ, Song J. Electrospray ionization-tandem mass spectrometry analysis of the mycolic acid profiles for the identification of common clinical isolates of mycobacterial species. J Microbiol Methods 2009;77:165-77.

Steingart KR, Henry M, Ng V, Hopewell PC, Ramsay A, Cunningham $\mathrm{J}$, et al. Fluorescence versus conventional sputum smear microscopy for tuberculosis: a systematic review. Lancet Infect Dis. 2006;6:57081.

Takayama K, Wang C, Besra GS. Pathway to synthesis and processing of mycolic acids in Mycobacterium tuberculosis. Clin Microbiol Rev 2005;18:81-101.

Toney NC, Toney SR, Butler WR. Utility of high-performance liquid chromatography analysis of mycolic acids and partial 16S rRNA gene sequencing for routine identification of Mycobacterium spp. in a national reference laboratory. Diagn Microbiol Infect Dis 2010;67:14352.

Tostmann A, Kik SV, Kalisvaart NA, Sebek MM, Verver S, Boeree MJ, van Soolingen D. Tuberculosis transmission by patients with smearnegative pulmonary tuberculosis in a large cohort in the Netherlands. Clin Infect Dis 2008;47:1135-42.

Viader-Salvadó JM, Molina-Torres CA, Guerrero-Olazarán M. Detection and identification of mycobacteria by mycolic acid analysis of sputum specimens and young cultures. J Microbiol Methods 2007;70:479-83

Vittor AY, Garland JM, Schlossberg D. Improving the diagnosis of tuberculosis: From QuantiFERON to new techniques to diagnose tuberculosis infections. Curr HIV/AIDS Rep 2011;8:153-63.

Wang J, Chen WF, Li QX. Rapid identification and classification of Mycobacterium spp. using whole-cell protein barcodes with matrix assisted laser desorption ionization time of flight mass spectrometry in comparison with multigene phylogenetic analysis. Anal Chim Acta 2012;716:133-7

World Health Organization. Global Tuberculosis Report 2012.

Yassin AF, Rainey FA, Brzezinka H, Burghardt J, Rifai M, Seifert P, Feldmann K, Schaal KP. Tsukamurella pulmonis sp. nov. Int J Syst Bacteriol. 1996;46(2):429-36.

Yuan Y, Zhu Y, Crane DD, Barry CE 3rd. The effect of oxygenated mycolic acid composition on cell wall function and macrophage growth in Mycobacterium tuberculosis. Mol Microbiol 1998;29:1449-58. 


\section{Rapid method for identification of Mycobacterium tuberculosis by use of electrospray ionization tandem mass spectrometry analysis of mycolic acids}

Szewczyk Rafał ${ }^{a, *}$, Kowalski Konrad $^{b}$, Janiszewska-Drobinska Beata ${ }^{c}$, Druszczynska Magdalena ${ }^{b}$

a, ${ }^{*}$ Corresponding author - Department of Biotechnology and Industrial Microbiology, Institute of Microbiology, Biotechnology and Immunology, Faculty of Biology and Environmental Protection, University of Łódź, Banacha 12/16, 90-237 Łódź, Poland, tel. 48426354460 , Fax. 484266558 18, rszewcz@biol.uni.lodz.pl

${ }^{b}$ Department of Immunology and Infectious Biology, Institute of Microbiology, Biotechnology and Immunology, Faculty of Biology and Environmental Protection, University of Lodz, Banacha 12/16, 90-237 Lodz, Poland,

${ }^{\mathrm{c}}$ Specialized Hospital of Tuberculosis, Lung Diseases and Rehabilitation, Szpitalna 5, 95-080 Tuszyn, Poland,

Contents:

Table S-1: p. S2

Table S-2: p. S3

Table S-1. Control microorganisms other that Mycobacterium checked for presence of mycolic acid using LC-MS/MS method.

\begin{tabular}{lll} 
Bacteria & Fungi & Yeast \\
\hline Corynebacterium glutamicum & Aspergillus nidulans & Schizoaccharomyces pombe \\
Rhodococcus equi & Cunninghamella elegans & Saccharomyces cerevisiae \\
Rhodococcus erythropolis & Metarhizium anisopliae & \\
Proteus vulgaris & \\
Escherichia coli & \\
Listeria inocua & \\
Klebsiella pneumoniae & \\
Arthrobacter sp. & \\
Pseudomonas fluorescens &
\end{tabular}

Table S-2. Summary of the clinical samples tests.

\begin{tabular}{|c|c|c|c|c|c|}
\hline \multirow[t]{2}{*}{ Group } & \multirow{2}{*}{$\begin{array}{l}\text { Material } \\
\text { number }\end{array}$} & \multirow{2}{*}{$\begin{array}{l}\text { AFB } \\
\text { smear } \\
\text { stain }\end{array}$} & \multirow{2}{*}{$\begin{array}{l}\text { Mtb } \\
\text { culture }\end{array}$} & \multicolumn{2}{|c|}{ LC-MS/MS analysis } \\
\hline & & & & direct & $\begin{array}{l}\text { after } 10 \text { days of } \\
\text { incubation }\end{array}$ \\
\hline \multirow{16}{*}{ TB patients } & 1 & positive & positive & positive & positive \\
\hline & 2 & positive & positive & positive & positive \\
\hline & 3 & positive & positive & positive & positive \\
\hline & 4 & positive & positive & positive & positive \\
\hline & 5 & positive & positive & positive & positive \\
\hline & 6 & positive & positive & positive & positive \\
\hline & 7 & positive & positive & positive & positive \\
\hline & 8 & positive & positive & positive & positive \\
\hline & 9 & positive & positive & positive & positive \\
\hline & 10 & negative & positive & positive & positive \\
\hline & 11 & negative & positive & positive & positive \\
\hline & 12 & negative & positive & negative & positive \\
\hline & 13 & negative & negative & negative & positive \\
\hline & 14 & negative & negative & unsure positive & positive \\
\hline & 15 & negative & negative & negative & positive \\
\hline & 16 & negative & positive & negative & negative \\
\hline \multirow{17}{*}{$\begin{array}{l}\text { Non-TB } \\
\text { patients }\end{array}$} & 17 & negative & negative & negative & negative \\
\hline & 18 & negative & negative & negative & negative \\
\hline & 19 & negative & negative & negative & negative \\
\hline & 20 & negative & negative & negative & negative \\
\hline & 21 & negative & negative & negative & negative \\
\hline & 22 & negative & negative & negative & negative \\
\hline & 23 & negative & negative & negative & negative \\
\hline & 24 & negative & negative & negative & negative \\
\hline & 25 & negative & negative & negative & negative \\
\hline & 26 & negative & negative & negative & negative \\
\hline & 27 & negative & negative & negative & negative \\
\hline & 28 & negative & negative & negative & negative \\
\hline & 29 & negative & negative & negative & negative \\
\hline & 30 & negative & negative & negative & negative \\
\hline & 31 & negative & negative & negative & negative \\
\hline & 32 & negative & negative & negative & negative \\
\hline & 33 & negative & negative & negative & negative \\
\hline \multirow{11}{*}{$\begin{array}{l}\text { Control } \\
\text { subjects }\end{array}$} & 34 & negative & negative & negative & negative \\
\hline & 35 & negative & negative & negative & negative \\
\hline & 36 & negative & negative & negative & negative \\
\hline & 37 & negative & negative & negative & negative \\
\hline & 38 & negative & negative & negative & negative \\
\hline & 39 & negative & negative & negative & negative \\
\hline & 40 & negative & negative & negative & negative \\
\hline & 41 & negative & negative & negative & negative \\
\hline & 42 & negative & negative & negative & negative \\
\hline & 43 & negative & negative & negative & negative \\
\hline & 44 & negative & negative & negative & negative \\
\hline
\end{tabular}

